\title{
Searching for the elusive critical endpoint at finite temperature and isospin density
}

D. K. Sinclair*

HEP Division, Argonne National Laboratory, 9700 South Cass Avenue, Argonne, IL 60439, USA

E-mail: dks@hep.anl.gov

\section{J. B. Kogut}

Department of Energy, Division of High Energy Physics, Washington, DC 20585, USA

and

Dept. of Physics - TQHN, Univ. of Maryland, 82 Regents Dr., College Park, MD 20742, USA

E-mail: jbkogut@umd.edu

\begin{abstract}
We consider 3-flavour lattice QCD with a finite chemical potential $\mu_{I}$ for isospin, close to the finite temperature transition from hadronic matter to a quark-gluon plasma. In this region one can argue that the position and probably the nature of this transition mimic those at finite quarknumber chemical potential $\mu$. The quark mass is chosen to be close to the critical mass at zero chemical potentials. Since the Binder cumulants used to determine the nature of this transition in $\operatorname{HMD}(\mathrm{R})$ simulations are very sensitive to the updating increment $d t$, we have switched to the newer exact RHMC algorithm for our simulations. Preliminary results indicate that there is no critical endpoint in the small $\mu_{I}$ regime, at least none connected with the critical point at zero chemical potentials.
\end{abstract}




\section{Introduction}

Direct simulations of QCD at fi nite baryon/quark number density are made diffi cult if not impossible because at fi nite quark-number chemical potential $\mu$ the fermion determinant is complex. At small chemical potentials, close to the fi nite temperature transition, various methods have been devised to circumvent this diffi culty, series expansions in $\mu$ [1, [, 目], analytic continuation from imaginary $\mu$ [月, 5, 6, 7, 8, 9], reweighting methods [10, 11] and canonical ensemble techniques [12, 13].

We adopt a different strategy, and simulate using the magnitude of the fermion determinant and ignoring the phase [14, 15]. This can be thought of as considering all quarks to be in isodoublets and introducing a fi nite chemical potential $\mu_{I}$ for isospin. In the region of small $\mu / \mu_{I}$, where the phase is expected to be less important one can argue that the fi nite $\mu$ and $\mu_{I}$ transitions might be identical. Since our fermion determinant is positive (or at least non-negative), we can use standard hybrid molecular-dynamics $\operatorname{HMD}(\mathrm{R})$ simulations [16]. However, for this algorithm, the Binder cumulants used to determine the nature of the fi nite temperature transition turn out to be strongly dependent on the updating increment $d t$. For this reason we now simulate using the rational hybrid monte-carlo (RHMC) algorithm [17, 18], which is exact in the sense of having no $d t$ dependence for observables.

In the low chemical potential domain, the most interesting feature expected in the phase diagram is the critical endpoint, where the fi nite temperature transition changes from a crossover to a fi rst-order transition as chemical potential is increased. The critical endpoint is expected to lie in the universality class of the 3-dimensional Ising model. For 3 flavours it had been expected that the critical point at zero chemical potentials, where the transition changes from a fi rst order transition to a crossover as mass is increased, would move to higher masses as the chemical potential increases, thereby becoming the critical endpoint. Our preliminary results indicate that this does not happen.

In section 2 we give the fermion action and make a few comments on the RHMC implementation. Section 3 gives our preliminary results. Our conclusions occupy section 4 .

\section{QCD at finite isospin density and the RHMC}

The pseudo-fermion action for QCD at fi nite $\mu_{l}$, used for the implementation of the RHMC algorithm is

$$
S_{p f}=p_{\psi}^{\dagger} \mathscr{M}^{-N_{f} / 8} p_{\psi}
$$

where $p_{\psi}$ is the momentum conjugate to the pseudo-fermion fi eld $\psi$.

$$
\mathscr{M}=\left[D\left(\frac{1}{2} \mu_{I}\right)+m\right]^{\dagger}\left[D\left(\frac{1}{2} \mu_{I}\right)+m\right]+\lambda^{2}
$$

is the quadratic Dirac operator, and we set $\lambda=0$ for our $\mu_{I}<m_{\pi}$ simulations.

To implement the RHMC method we need to know positive upper and lower bounds to the spectrum of $\mathscr{M} .25$ exceeds the upper bound for the $\mu_{I}$ range of interest. We use a speculative lower bound of $10^{-4}$ since the actual lower bound of the spectrum is unknown. This is justifi ed by varying the choice of lower bounds and comparing the results [19]. For $N_{f}=3$ we use a 
$(20,20)$ rational approximation to $\mathscr{M}^{( \pm 3 / 16)}$ at the ends of each trajectory, and a $(10,10)$ rational approximation to $\mathscr{M}^{(-3 / 8)}$ for the updating.

\section{Simulations and Results}

We are simulating lattice QCD with staggered fermions and $N_{f}=3$ at quark masses close to $m_{c}$, the critical mass for $\mu=\mu_{I}=0$ on $8^{3} \times 4,12^{3} \times 4$ and $16^{3} \times 4$ lattices. $m=0.02,0.025,0.03$, 0.035 , and $\mu_{I}=0.0,0.2,0.3$. For our $12^{3} \times 4$ simulations we use runs of 300,000 trajectories at each of $4 \beta$ values close to $\beta_{c}$, for each $m$ and $\mu_{I}$. We mostly use $d t=0.05$ for which length-1 trajectories give acceptances of $\sim 70 \%$ for the RHMC algorithm.

To determine the nature of the transition, we use 4-th order Binder cumulants [20] for the chiral condensate. For any observable $X$ this cumulant is defi ned by

$$
B_{4}(X)=\frac{\left\langle(X-\langle X\rangle)^{4}\right\rangle}{\left\langle(X-\langle X\rangle)^{2}\right\rangle^{2}}
$$

where the $X \mathrm{~s}$ are lattice averaged quantities. For infi nite volumes, $B_{4}=3$ for a crossover, $B_{4}=1$ for a fi rst-order transition and $B_{4}=1.604(1)$ for the 3-dimensional Ising model. Thus, if there is a critical endpoint we would expect $B_{4}$ to decrease with increasing $\mu_{I}$, passing through a value close to the Ising value at the critical $\mu_{I}$.

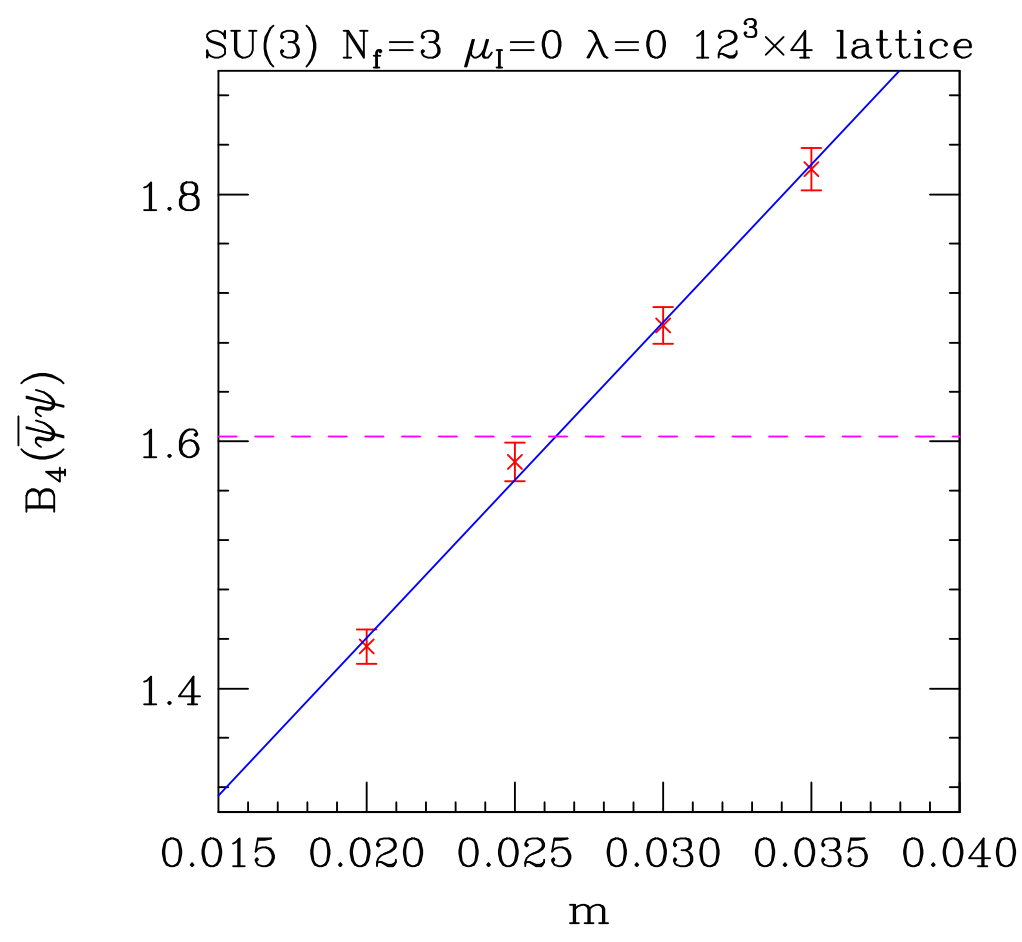

Figure 1: Binder cumulant at $T=T_{c}$ as a function of mass at $\mu_{I}=\mu=0$.

Figure 1 shows our preliminary measurements of the Binder cumulant for the chiral condensate as a function of mass at $\mu_{I}=\mu=0$ from our $12^{3} \times 4$ simulations. Taking the point where the straight-line fi t passes through the Ising value as our estimate for the critical mass yields $m_{c}=$ 
0.0264(3). Each of the points in this graph were obtained by averaging the Binder cumulants taken from several $\beta$ values close to the transition, and extrapolated to $\beta_{c}$ which minimizes these cumulants, using Ferrenberg-Swendsen rewieghting [21].

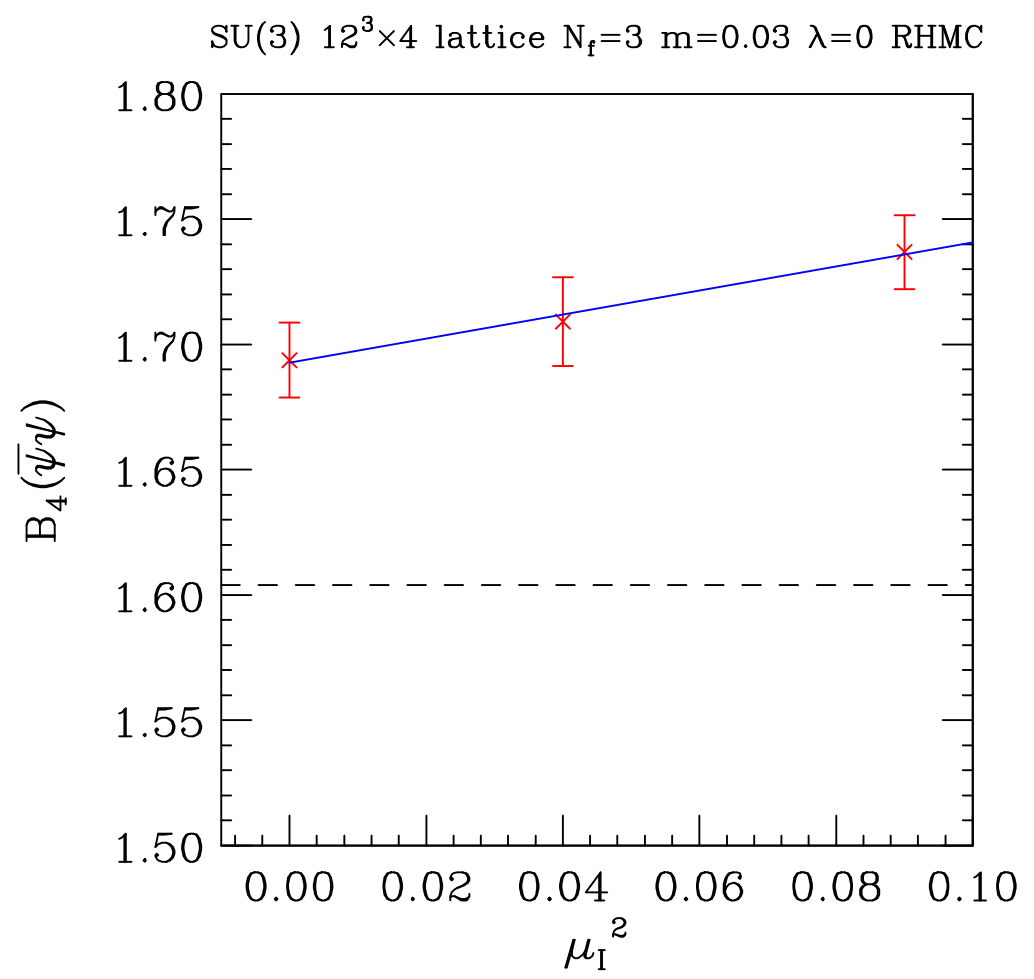

Figure 2: Binder cumulant at $T=T_{c}$ as a function of $\mu_{I}^{2}$ at $m=0.03$.

The $\mu_{I}$ dependence of this Binder cumulant at $\beta_{c}\left(\mu_{I}\right)$ is shown in fi gure 8 , for $m=0.03$, a little above $m_{c}$. It is clear that, rather than decreasing with increasing $\mu_{I}$, it actually increases slowly. Since $\beta_{c}$ and hence $T_{c}$ decrease with increasing $\mu_{I}$, in physical units $m$ is actually decreasing with increasing $\mu_{I}$ meaning that at fi xed physical $m$ the rise would be even more pronounced. The behaviour at $m=0.035$ is very similar.

Figure 3 shows the dependence of the transition $\beta, \beta_{c}$, on $\mu_{I}$. As mentioned above, $\beta_{c}$ and hence the transition temperature $T_{c}$ fall (slowly) with increasing $\mu_{I}$ as expected. The fi ts shown to this preliminary 'data' are:

$$
\begin{array}{ll}
\beta_{c}=5.15326(10)-0.173(2) \mu_{I}^{2} & m=0.035 \\
\beta_{c}=5.14386(8)-0.172(1) \mu_{I}^{2} & m=0.030 \\
\beta_{c}=5.13426(12)-0.179(4) \mu_{I}^{2} & m=0.025
\end{array}
$$

which is in reasonable agreement with the results of de Forcrand and Philipsen for the $\mu$ dependence of the transition temperature, obtained from analytic continuation from imaginary $\mu$ if we make the identifi cation $\mu_{I}=2 \mu$.

Figure 7 shows the $d t$ dependence of the Binder cumulants at the transition for $m=0.035$, $\mu_{I}=0.2$ in the $\operatorname{HMD}(\mathrm{R})$ simulations. The exact RHMC result, which has no $d t$ dependence, is 


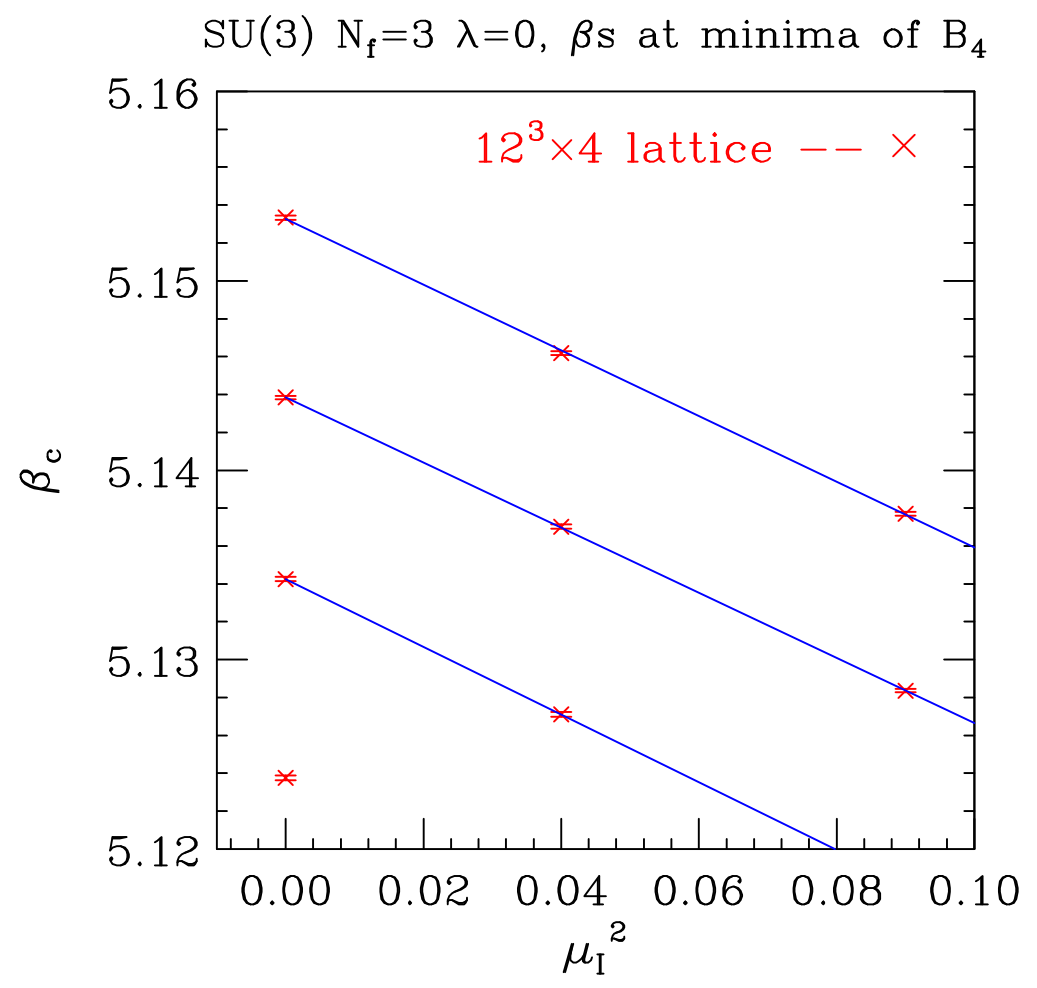

Figure 3: $\beta_{c}$ as functions of $\mu_{I}^{2}$. From top to bottom, $m=0.035, m=0.03, m=0.025, m=0.02$.

plotted on this graph at $d t=0$. It is clear that the RHMC result is consistent with the $d t \rightarrow 0$ limit of the $\operatorname{HMD}(\mathrm{R})$ results. The actual value of $d t$ used in the RHMC simulations was $d t=0.05$, the value of $d t$ for the rightmost point on this graph, showing one advantage of using this new algorithm.

\section{Conclusions}

We simulate lattice QCD with 3 flavours of staggered quarks with a small chemical potential $\mu_{I}<m_{\pi}$ for isospin, in the neighbourhood of the fi nite temperature transition from hadronic matter to a quark-gluon plasma. Fourth order Binder cumulants are used to probe the nature of this transition and search for the critical endpoint for masses slightly above the critical mass for zero chemical potentials. Earlier simulations using the $\operatorname{HMD}(\mathrm{R})$ algorithm were plagued by large fi nite $d t$ errors [15]. We now use the RHMC algorithm which is exact in the sense of having no fi nite $d t$ errors.

We measure the critical mass to be $m_{c}=0.0264(3)$ for $N_{t}=4$, in agreement with the recent results of de Forcrand and Philipsen [5], but considerably below earlier measurements which found values close to $m_{c}=0.033$ [22, 23, 24]. These higher values were due to using the HMD(R) algorithm with $d t$ large enough to produce large systematic errors.

For masses greater than $m_{c}$ we found that the Binder cumulant for the chiral condensate increases with increasing $\mu_{I}$ and thus shows no evidence for a critical endpoint, contrary to earlier expectations. This also agrees with the observations of de Forcrand and Philipsen [巨] for fi nite $\mu$, 


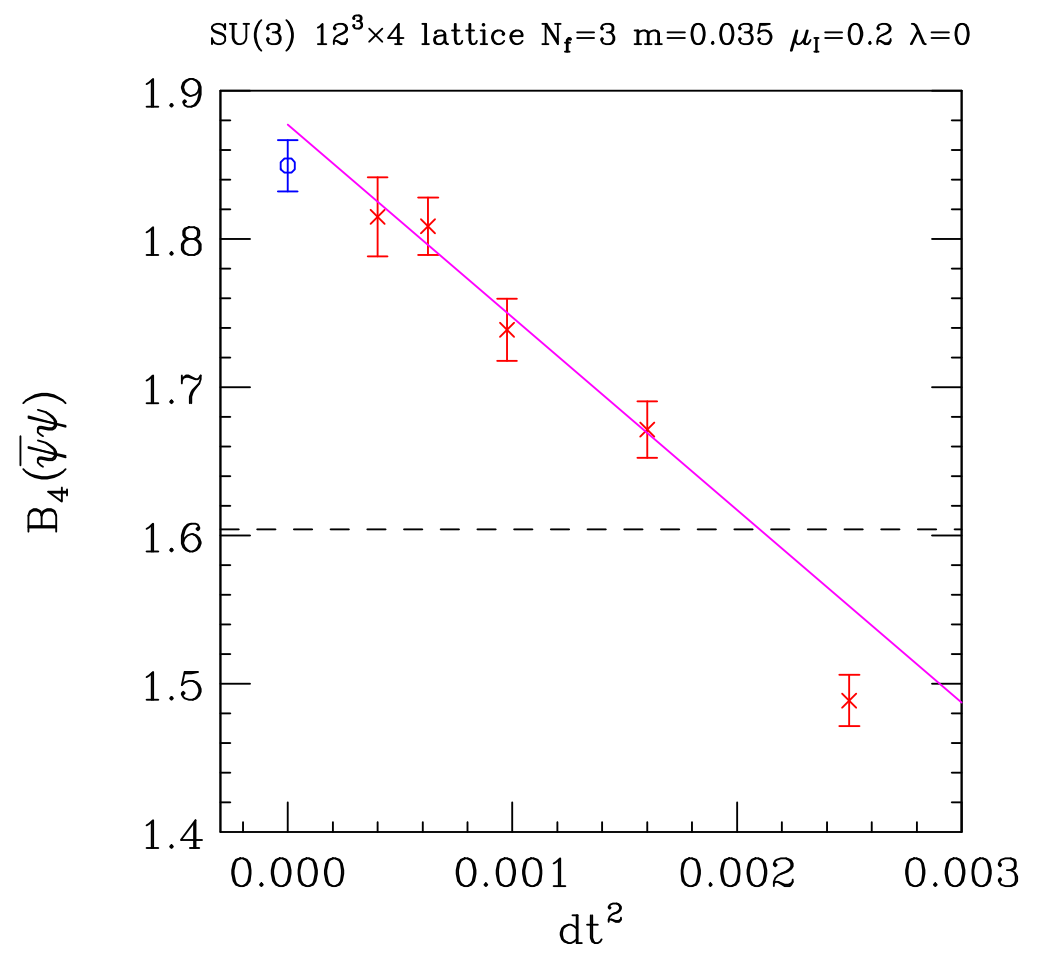

Figure 4: Binder cumulants for $m=0.035, \mu_{I}=0.2$ for $\operatorname{HMD}(\mathrm{R})$ simulations as a function of $d t^{2}$ compared with that from RHMC simulations.

emphasizing the similarities between fi nite $\mu$ and fi nite $\mu$ for small $\mu, \mu_{I}$, near the fi nite temperature transition. On these relatively small lattices $\left(12^{3} \times 4\right)$, we really should minimize the Binder cumulant of linear combinations of the chiral condensate, the plaquette and the isospin density to obtain the desired eigenfi eld of the renormalization group equations, to draw reliable conclusions [22] ${ }^{1}$.

We end with the observation that we have used RHMC simulations where we do not know a positive lower bound for the spectrum of the quadratic Dirac operator. This is done by choosing a speculative lower bound and justifying our choice a postiori. We refer the reader to our recent paper on this subject [19].

\section{Acknowledgements}

JBK is supported in part by a National Science Foundation grant NSF PHY03-04252. DKS is supported by the U.S. Department of Energy, Division of High Energy Physics, Contract W-31109-ENG-38. We thank Philippe de Forcrand and Owe Philipsen for helpful discussions. Simulations are performed on Jazz at Argonne, Tungsten and Cobalt at NCSA, Bassi and Jacquard at NERSC, DataStar at SDSC and PC's in Argonne HEP.

\section{References}

[1] C. R. Allton et al., Phys. Rev. D 66, 074507 (2002) [arXiv:hep-lat/0204010].

\footnotetext{
${ }^{1}$ We thank Frithjof Karsch for reminding us of this fact
} 
[2] C. R. Allton et al., Phys. Rev. D 71, 054508 (2005) [arXiv:hep-lat/0501030].

[3] R. V. Gavai and S. Gupta, Phys. Rev. D 68, 034506 (2003) [arXiv:hep-lat/0303013].

[4] P. de Forcrand and O. Philipsen, Nucl. Phys. B 642, 290 (2002) [arXiv:hep-lat/0205016].

[5] P. de Forcrand and O. Philipsen, arXiv:hep-lat/0607017.

[6] V. Azcoiti, G. Di Carlo, A. Galante and V. Laliena, JHEP 0412, 010 (2004) [arXiv:hep-lat/0409157].

[7] V. Azcoiti, G. Di Carlo, A. Galante and V. Laliena, Nucl. Phys. B 723, 77 (2005) [arXiv:hep-lat/0503010].

[8] M. D'Elia and M. P. Lombardo, Phys. Rev. D 67, 014505 (2003) [arXiv:hep-lat/0209146].

[9] M. D'Elia and M. P. Lombardo, Phys. Rev. D 70, 074509 (2004) [arXiv:hep-lat/0406012].

[10] Z. Fodor and S. D. Katz, Phys. Lett. B 534, 87 (2002) [arXiv:hep-lat/0104001].

[11] Z. Fodor and S. D. Katz, JHEP 0404, 050 (2004) [arXiv:hep-lat/0402006].

[12] S. Kratochvila and P. de Forcrand, PoS LAT2005, 167 (2006) [arXiv:hep-lat/0509143].

[13] A. Alexandru, M. Faber, I. Horvath and K. F. Liu, Phys. Rev. D 72, 114513 (2005) [arXiv:hep-lat/0507020].

[14] J. B. Kogut and D. K. Sinclair, Phys. Rev. D 66, 034505 (2002) [arXiv:hep-lat/0202028].

[15] J. B. Kogut and D. K. Sinclair, arXiv:hep-lat/0509095.

[16] S. A. Gottlieb, W. Liu, D. Toussaint, R. L. Renken and R. L. Sugar, Phys. Rev. D 35, 2531 (1987).

[17] A. D. Kennedy, I. Horvath and S. Sint, Nucl. Phys. Proc. Suppl. 73, 834 (1999) [arXiv:hep-lat/9809092].

[18] M. A. Clark, P. de Forcrand and A. D. Kennedy, PoS LAT2005, 115 (2006) [arXiv:hep-lat/0510004].

[19] J. B. Kogut and D. K. Sinclair, arXiv:hep-lat/0608017.

[20] K. Binder, Z. Phys. B 43, 119 (1981).

[21] A. M. Ferrenberg and R. H. Swendsen, Phys. Rev. Lett. 61, 2635 (1988).

[22] F. Karsch, E. Laermann and C. Schmidt, Phys. Lett. B 520, 41 (2001) [arXiv:hep-lat/0107020].

[23] N. H. Christ and X. Liao, Nucl. Phys. Proc. Suppl. 119, 514 (2003).

[24] P. de Forcrand and O. Philipsen, Nucl. Phys. B 673, 170 (2003) [arXiv:hep-lat/0307020]. 